\title{
Validation of the Puumala virus rapid field test for bank voles in Germany
}

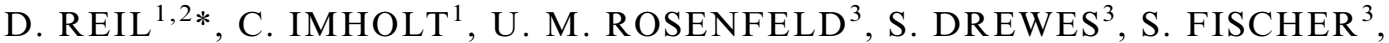 \\ E. HEUSER ${ }^{3}$, R. PETRAITYTE-BURNEIKIENE ${ }^{4}$, R. G. ULRICH ${ }^{3}$ AND J. JACOB ${ }^{1}$ \\ ${ }^{1}$ Julius Kühn-Institute, Federal Research Centre for Cultivated Plants, Institute for Plant Protection in \\ Horticulture and Forests, Vertebrate Research, Münster, Germany \\ ${ }^{2}$ University of Potsdam, Institute of Biochemistry and Biology, Animal Ecology, Potsdam, Germany \\ ${ }^{3}$ Friedrich-Loeffler-Institut, Federal Research Institute for Animal Health, Institute for Novel and Emerging \\ Infectious Diseases, Greifswald-Insel Riems, Germany \\ ${ }^{4}$ Vilnius University, Institute of Biotechnology, Vilnius, Lithuania
}

Received 31 May 2016; Final revision 24 August 2016; Accepted 5 October 2016; first published online 3 November 2016

\section{SUMMARY}

Puumala virus (PUUV) causes many human infections in large parts of Europe and can lead to mild to moderate disease. The bank vole (Myodes glareolus) is the only reservoir of PUUV in Central Europe. A commercial PUUV rapid field test for rodents was validated for bank-vole blood samples collected in two PUUV-endemic regions in Germany (North Rhine-Westphalia and Baden-Württemberg). A comparison of the results of the rapid field test and standard ELISAs indicated a test efficacy of 93-95\%, largely independent of the origin of the antigens used in the ELISA. In ELISAs, reactivity for the German PUUV strain was higher compared to the Swedish strain but not compared to the Finnish strain, which was used for the rapid field test. In conclusion, the use of the rapid field test can facilitate short-term estimation of PUUV seroprevalence in bank-vole populations in Germany and can aid in assessing human PUUV infection risk.

Key words: Antibody detection, early warning, Europe, hantavirus, Myodes glareolus.

\section{INTRODUCTION}

There is an increasing number of hantaviruses documented in Europe [1-3] and other parts of the world [4]. All European hantaviruses are thought to be associated with small mammal hosts such as rodents and shrews [4]. PUUV seems to be the predominant hantavirus species in Europe, which is possibly due to the wide distribution of its principal host, the bank vole

\footnotetext{
* Author for correspondence: Mrs D. Reil, Julius Kühn-Institute, Federal Research Centre for Cultivated Plants, Institute for Plant Protection in Horticulture and Forests, Vertebrate Research, Münster, Germany.

(Email: daniela.reil@julius-kuehn.de)
}

(Myodes glareolus) [3]. It is one of the hantaviruses that cause clinical symptoms in humans [5].

A PUUV infection can cause a mild to moderate form of haemorrhagic fever with renal syndrome in humans, designated nephropathia epidemica (NE) [5]. In Germany, an annual maximum of $>2000$ human infections has been reported since the disease was declared notifiable in 2001 (SurvStat@RKI 2.0, https://survstat.rki.de). In other European countries such as Finland and Sweden as high as $>3000$ cases were recorded in peak years [6].

In temperate Europe, human NE outbreaks are related to beech (Fagus sylvatica) mast that promotes population outbreaks of bank voles [7-9] due to 
enhanced food supply and resulting increased survival and reproduction (bottom-up regulation). In contrast, bank-vole population dynamics in Northern Europe are rather predator driven (top-down regulation) [10]. Beech mast seems to be closely related to climate patterns [11] and has occurred in the last 15 years about every 2-3 years in Central Europe [7, 12]. In years with high bank-vole density more PUUVinfected bank voles are present [13] and more people become infected [7].

The close relationship of beech mast and bank-vole population dynamics and the effect of the latter on human PUUV infection allow weather-based prediction of the general risk of human PUUV infection. Predictive models can also utilize tree seed mast $[8,14]$, rodent host abundance $[15,16]$, other environmental parameters such as vegetation or land use [17, 18] and recent methodological progress [19]. Weather-based models have been developed and provide a robust baseline for alerting health officials and high-risk groups such as forestry workers if a substantially increased infection risk is expected $[11,20]$.

However, such a predictive approach requires information about the presence of PUUV in regional bankvole populations because high bank-vole abundance only in combination with the presence of PUUV in the rodent host population elevates the infection risk for humans. Information about PUUV seroprevalence in the host population should be reliable, quick to obtain and based on a non-invasive sample collection. Rapid field tests have been designed to be used in the field to quickly screen rodents for the presence of pathogen-specific antibodies [21]. They provide the necessary data faster than standard diagnostic approaches such as enzyme linked immunosorbent assay (ELISA) for the detection of PUUV-specific antibodies or genetic analyses for the detection of viral genome. In a study by Sirola et al. [21] such a test (Reagena, Finland) has been validated for bank voles in Finland and resulted in 100\% efficacy. This test is based on the PUUV prototype strain from Finland, Sotkamo (SOT [22, 23]). No rapid test exists for German PUUV strains nor have the existing tests been systematically validated for German bank voles. In a pilot laboratory study of German bank voles [24] 15 of 18 rapid field test results matched ELISA and Western blot test results using a recombinant antigen of a Swedish PUUV strain (Vranica/Hällnäs, VRA $[25,26])$. As test efficacy seems to vary according to geographical origin of the vole samples and the antigens used we aimed to evaluate results of the rapid field test regarding its performance in detecting PUUV-specific antibodies in a field trial with bank voles in Germany. Vole blood samples were screened with the rapid field test and analysed with in-house ELISAs using antigens of SOT, a German strain and the VRA strain.

\section{MATERIALS AND METHODS}

Bank-vole populations were sampled 2013-2015 in North Rhine-Westphalia (NW) and BadenWürttemberg (BW) because PUUV is present in both federal states [27]. Voles were trapped 2-3 times a year, in spring, summer and autumn in forest sites close to Billerbeck $\left(51^{\circ} 59^{\prime} 38^{\prime \prime} \mathrm{N}, 7^{\circ} 18^{\prime} 59^{\prime \prime} \mathrm{E}\right)$ in NW and near Weissach $\left(48^{\circ} 49^{\prime} 47^{\prime \prime} \mathrm{N}, 8^{\circ} 57^{\prime} 58^{\prime \prime} \mathrm{E}\right)$ in BW. Bank voles were captured using 49 Ugglan live-traps (Grahnab, Sweden) set in $10 \times 10 \mathrm{~m}$ grids and a blood sample of $50 \mu \mathrm{l}$ was collected from each bank vole from the retro-orbital sinus or facial vein using capillary pipettes $\left(50 \mu \mathrm{l}\right.$; Ringcaps ${ }^{\circledR}$, Germany). Ten microlitres of the blood samples were analysed directly after trapping using the rapid field test for rodents (ReaScan ${ }^{\circledR}$ Ab-Dect Puumala IgG, Reagena, Finland). The diluted blood sample (mixed with IgG-coated gold particles) was transferred into a test cassette where the sample flows across a membrane with bounded PUUV antigen. The ReaScan Reader is designed to display a reading of a value directly proportional to the concentration of PUUV-specific antibodies captured by the membrane (ReaScan Ab-Dect Puumala IgG, Instructions for use version 1.2). Definition of reactivity followed the manufacturer's protocol: cut-off values were $<5$ (negative), 5-15 (equivocal) and $>15$ (positive). However, equivocal results were counted as positive for further analyses due to a measurable membrane reaction. Testing was done in a car or nearby building.

The remaining $40 \mu \mathrm{l}$ of the blood samples were stored at $-20^{\circ} \mathrm{C}$ until analyses with in-house ELISA (for details see Mertens et al. [28]), which were done using yeast-expressed recombinant antigens of PUUV strains from Bavaria, Germany (BAWA [29]), Sweden (VRA) and the homologous SOT strain.

All samples were initially analysed using the German BAWA antigen ( $n=341$ samples). Additional testing was performed using VRA $(n=104$ samples), which was replaced by SOT ( $n=237$ samples) as soon as SOT was available. Due to restrictions in sample volume not all samples could be tested with all 
Table 1. Contingency table (McNemar test) for validation of rapid field test and in-house ELISAs for detection of $P U U V$-specific antibodies in bank voles

\begin{tabular}{llll}
\hline \hline Rapid field test & $\begin{array}{l}\text { ELISA positive } \\
\text { (BAWA/VRA/SOT) }\end{array}$ & $\begin{array}{l}\text { ELISA negative } \\
\text { (BAWA/VRA/SOT) }\end{array}$ & $\begin{array}{l}\text { Total } \\
\text { (BAWA/VRA/SOT) }\end{array}$ \\
\hline Positive & $n=31 / 9 / 22$ & $n=3 / 0 / 3$ & $n=34 / 9 / 25$ \\
& A: true positive & B: false positive & $n=303 / 94 / 209$ \\
Negative & $n=4 / 1 / 3$ & D: true negative & $n=307 / 95 / 212$ \\
& C: false negative & $n=306 / 94 / 212$ & $n=341 / 104 / 237$ \\
\hline
\end{tabular}

PUUV antigens used for in-house ELISA: BAWA, German strain Bavaria; VRA, Swedish strain Vranica/Hällnäs; SOT, Finnish strain Sotkamo.

antigens. Equivocal results were regarded positive for further evaluation.

Sensitivity and specificity of the rapid field test compared to the in-house ELISAs were calculated based on the contingency table of the McNemar test. Test efficacy was estimated by calculating the mean of sensitivity and specificity. Optical density determined in ELISAs of positive (and equivocal) samples was compared between BAWA and VRA ( $n=10$ samples) as well as BAWA and SOT ( $n=25$ samples) antigens. Data were analysed with a $t$ test using SPSS Statistics for Windows v. 22.0 (IBM Corp., USA).

\section{RESULTS}

In NW and BW 111 and 230 bank voles, respectively (total 341) were trapped and blood samples collected. In the rapid field test, 23/341 bank voles were PUUV positive and test results of 11 bank voles were equivocal. As described above, equivocal results of the rapid field test were regarded positive even if the cut-off value for PUUV positive evaluation was not exceeded because a measurable test membrane reaction was recorded. Further, this decision is supported by equivocal results of the rapid field tests corresponding to positive test results in ELISA in $82 \%$ of the cases. Hence, we regarded 34/341 animals to be seropositive resulting in a seroprevalence of $10 \%$ (Table 1).

In the in-house ELISAs, 35/341 (10\%) samples were positive using BAWA, 10/104 (10\%) samples using VRA and 25/237 (11\%) samples using SOT (Table 1). Thus, PUUV detection using the rapid field test resulted in 7\% false positives and 1\% false negatives compared to ELISA (mean values in comparison to all three antigens) (Table 2).

Depending on the PUUV strain used in the ELISA rapid test sensitivity was $88-90 \%$ and specificity
Table 2. Evaluation of results of the antibody detection by rapid field test and in-house ELISAs using PUUV strains BAWA, VRA and SOT

\begin{tabular}{lllll}
\hline \hline & & BAWA & VRA & SOT \\
\hline Sensitivity X & A/(A + C) & $88 \cdot 6 \%$ & $90 \cdot 0 \%$ & $88 \cdot 0 \%$ \\
Specificity Y & D/(B + D) & $99 \cdot 0 \%$ & $100 \cdot 0 \%$ & $98 \cdot 6 \%$ \\
Efficacy & (X+ Y)/2 & $93 \cdot 8 \%$ & $95 \cdot 0 \%$ & $93 \cdot 3 \%$ \\
\hline \hline
\end{tabular}

BAWA, German strain Bavaria; VRA, Swedish strain Vranica/Hällnäs; SOT, Finnish strain Sotkamo.

Letters A, B, C, D refer to Table 1.

$99-100 \%$. Test efficacy varied slightly between $93 \%$ and $95 \%$ according to the PUUV strain used.

The comparison of ELISA test results for antiPUUV-positive bank voles demonstrated $>50 \%$ higher optical density values for BAWA compared to VRA antigen $\left(T_{9}=2 \cdot 88, P=0 \cdot 02\right)$. In contrast, values were similar for BAWA and SOT antigens $\left(T_{24}=1.90, P>0.05\right)$ (Fig. 1).

\section{DISCUSSION}

Serological PUUV testing of bank voles is needed for scientific investigations as well as for surveillance and public health-related measures. For all these applications, quick and reliable test results are highly beneficial. In this regard, the rapid field test considered here is a suitable tool for German PUUV strains although the test is based on an antigen of the Finnish SOT PUUV strain.

Test efficacy of the rapid field test (93-95\%) was similar to the in-house ELISAs, independent on the recombinant antigen used. This is adequate for most purposes of rapid PUUV detection in the field. However, there were slight differences in the reactivity of antigens used in ELISAs (BAWA, VRA, SOT) with 


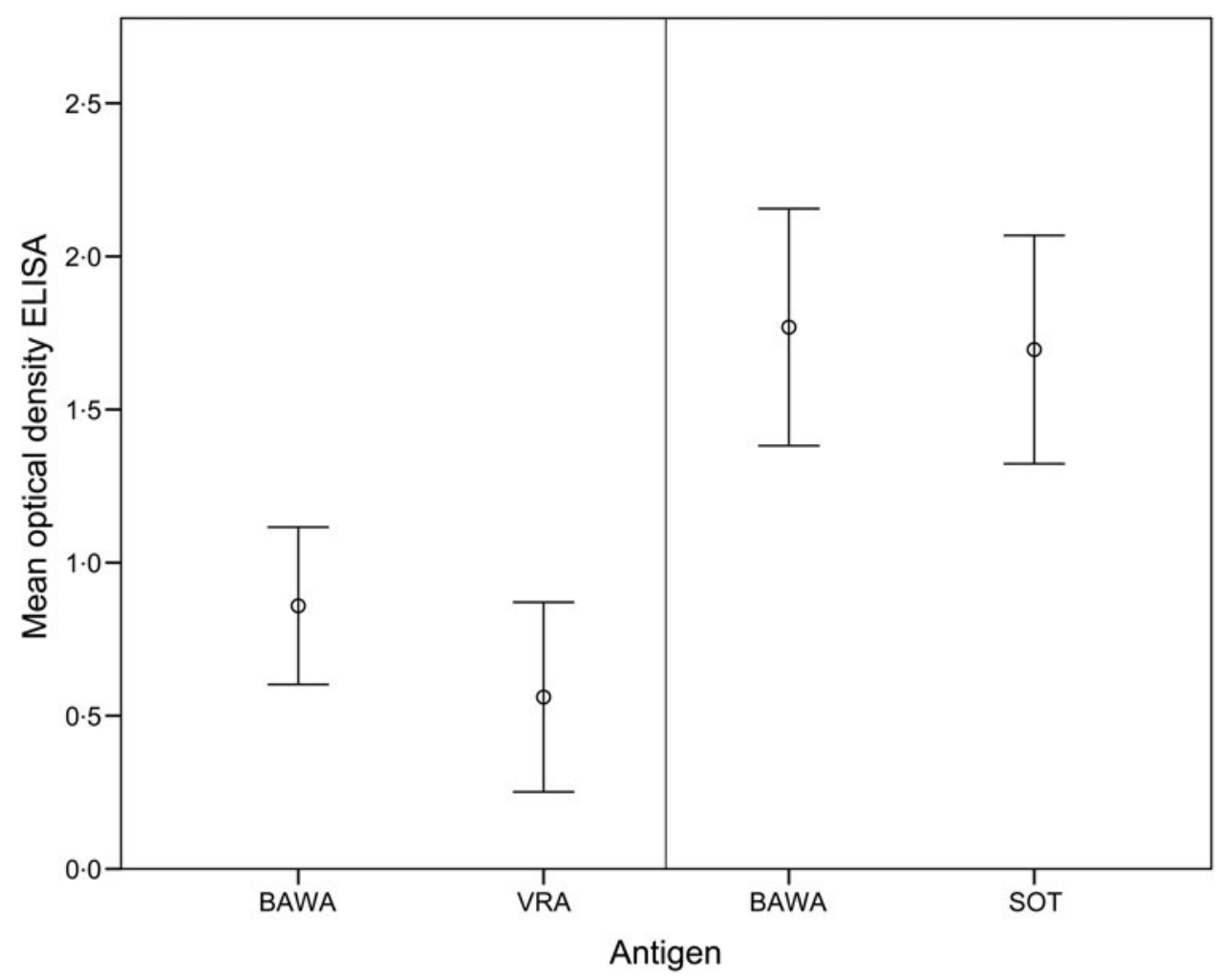

Fig. 1. Mean optical density values of the ELISA for detection of PUUV-specific antibodies in bank voles based on positive (and equivocal) samples analysed with antigens BAWA and VRA ( $n=10$ samples) as well as BAWA and SOT ( $n=25$ samples). BAWA, German strain Bavaria; VRA, Swedish strain Vranica/Hällnäs; SOT, Finnish strain Sotkamo.

blood samples from bank voles in Germany. This may be due to geographical and hence genetic variation of PUUV strains from Finland, Sweden and Germany. Optical density values in ELISAs were almost identical for SOT but considerably lower for VRA compared to values obtained using antigen from the German BAWA strain. This supports a reliable application of the Finnish rapid field test for German bankvole samples.

Sirola et al. [21] validated the rapid field test with 105 fresh blood samples collected in Finland at a seroprevalence of $15 \%$ and reported $100 \%$ sensitivity, specificity and efficacy. The slightly better performance of the rapid field test in their study is most probably due to the perfect match of Finnish bank-vole blood samples and the rapid field test based on the Finnish PUUV strain.

Despite the rapid field test failing to achieve optimal results, PUUV antibodies can be sufficiently detected in field conditions. The use of a rapid field test would facilitate short-term estimation of PUUV seroprevalence in bank-vole populations because laboratory assays testing for the presence of PUUVspecific antibodies or genome are significantly more time consuming and require costly specialized equipment. The rapid field test allows results to be obtained within minutes. The application of the test can be based on a non-invasive sample collection without sacrificing animals, because only a small blood volume $(10 \mu \mathrm{l})$ is needed for testing. This is not only relevant for optimizing study design in, e.g. long-term monitoring but also for improved animal ethics.

We regarded equivocal test results in the rapid field test and ELISA as positives because in future application of the rapid field test for assessing PUUV presence in bank voles for human health, producing false positives is more acceptable than false negatives.

In conclusion, the use of the rapid field test can aid health authorities in the future to quickly assess the current risk of human PUUV infection based on PUUV seroprevalence in the rodent reservoir in a timely and cost-effective manner. Monitoring strategies for rodents in Germany have recently been published by Jacob et al. [30]. In addition, harmonized methods for rodent monitoring and diagnostic routines are publicly available via 'species cards' (http://www. aphaea.eu/cards/species/voles) and 'diagnostic cards' (http://www.aphaea.eu/cards/diagnosis/hantavirosis). 
This information combined with the use of the rapid field test is useful in providing the data necessary to predict human PUUV infection risk temporally and spatially to support early warnings and preventative action.

\section{ACKNOWLEDGEMENTS}

We thank Dörte Kaufmann for technical assistance. Animal experimentation was approved by state authorities (North Rhine-Westphalia: 8.87-51.05.20.09.210 and 84-02.04.2014.A227, Baden-Württemberg 359185.82/0261). This study was commissioned and funded by the Federal Environment Agency (UBA) within the Environment Research Plan of the German Federal Ministry for the Environment, Nature Conservation, Building and Nuclear Safety (BMUB) (grant no. 371348 401).

\section{DECLARATION OF INTEREST}

None.

\section{REFERENCES}

1. Vapalahti O, et al. Hantavirus infections in Europe. Lancet Infectious Diseases 2003; 3: 653-661.

2. Klempa B, Radosa L, Kruger DH. The broad spectrum of hantaviruses and their hosts in Central Europe. Acta Virologica 2013; 57: 130-137.

3. Heyman P, et al. A five-year perspective on the situation of haemorrhagic fever with renal syndrome and status of the hantavirus reservoirs in Europe, 2005-2010. Eurosurveillance 2011; 16: 15-22.

4. Schlegel M, et al. Hantavirus emergence in rodents, insectivores and bats: what comes next? In: Johnson $\mathrm{N}$, ed. The Role of Animals in Emerging Viral Diseases. Boston: Academic Press, 2014, pp. 235-292.

5. Settergren B. Clinical aspects of nephropathia epidemica (Puumala virus infection) in Europe: a review. Scandinavian Journal of Infectious Diseases 2000; 32: 125-132.

6. Vaheri A, et al. Hantavirus infections in Europe and their impact on public health. Reviews in Medical Virology 2013; 23: 35-49.

7. Reil D, et al. Beech fructification and bank vole population dynamics-combined analyses of promoters of human Puumala virus infections in Germany. PLoS ONE 2015; 10: 1-14.

8. Clement $\mathbf{J}$, et al. Relating increasing hantavirus incidences to the changing climate: the mast connection. International Journal of Health Geographics 2009; 8: $1-11$.

9. Selva N, et al. Mast pulses shape trophic interactions between fluctuating rodent populations in a primeval forest. PLoS ONE 2012; 7.
10. Hanski I, et al. Small-rodent dynamics and predation. Ecology 2001; 82: 1505-1520.

11. Imholt C, et al. Quantifying the past and future impact of climate on outbreak patterns of bank voles (Myodes glareolus). Pest Management Science 2015; 71: 166-172.

12. Hansson L, Jedrzejewska B, Jedrzejewski W. Regional differences in dynamics of bank vole populations in Europe. Polish Journal of Ecology 2000; 48: 163-177.

13. Escutenaire S, et al. Spatial and temporal dynamics of Puumala hantavirus infection in red bank vole (Clethrionomys glareolus) populations in Belgium. Virus Research 2000; 67: 91-107.

14. Tersago K, et al. Hantavirus disease (nephropathia epidemica) in Belgium: effects of tree seed production and climate. Epidemiology and Infection 2009; 137: 250-256.

15. Kallio ER, et al. Cyclic hantavirus epidemics in humans-predicted by rodent host dynamics. Epidemics 2009; 1: 101-107.

16. Olsson GE, et al. Predicting high risk for human hantavirus infections, Sweden. Emerging Infectious Diseases 2009; 15: 104-106.

17. Zeimes CB, et al. Landscape and regional environmental analysis of the spatial distribution of hantavirus human cases in Europe. Frontiers in Public Health 2015; 3.

18. Amirpour Haredasht S, et al. Model-based prediction of nephropathia epidemica outbreaks based on climatological and vegetation data and bank vole population dynamics. Zoonoses and Public Health 2013; 60: 461-477.

19. Zeimes CB, et al. Modelling zoonotic diseases in humans: comparison of methods for hantavirus in Sweden. International Journal of Health Geographics 2012; 11.

20. Reil D, et al. Environmental conditions in favour of a hantavirus outbreak in 2015 in Germany? Zoonoses and Public Health 2016; 63: 83-88.

21. Sirola H, et al. Rapid field test for detection of hantavirus antibodies in rodents. Epidemiology and Infection 2004; 132: 549-553.

22. Brummer-Korvenkontio M, Henttonen H, Vaheri A. Hemorrhagic fever with renal syndrome in Finland: ecology and virology of nephropathia epidemica. Scandinavien Journal of Infectious Diseases 1982; 36: 88-91.

23. Razanskiene A, et al. High yields of stable and highly pure nucleocapsid proteins of different hantaviruses can be generated in the yeast Saccharomyces cerevisiae. Journal of Biotechnology 2004; 111: 319-333.

24. Essbauer SS, et al. Elucidation of unusual disease outbreaks: the outbreak of Puumala virus-related nephropathia epidemica in a German metropolis [in German]. Wehrmedizinische Monatsschriften 2007; 51: $325-329$.

25. Dargeviciute A, et al. Yeast-expressed Puumala hantavirus nucleocapsid protein induces protection in a bank vole model. Vaccine 2002; 20: 3523-3531.

26. Reip A, et al. Coding strategy of the $\mathrm{S}$ and $\mathrm{M}$ genomic segments of a hantavirus representing a new subtype of 
the Puumala serotype. Archives of Virology 1995; 140: 2011-2026.

27. Hofmann J, et al. Hantavirus outbreak, Germany, 2007. Emerging Infectious Diseases 2008; 14: 850-852.

28. Mertens M, et al. Seroepidemiological study in a Puumala virus outbreak area in South-East Germany. Medical Microbiology and Immunology 2009; 198: 83-91.
29. Mertens M, et al. Phylogenetic analysis of Puumala virus subtype Bavaria, characterization and diagnostic use of its recombinant nucleocapsid protein. Virus Genes 2011; 43: 177-191.

30. Jacob J, et al. Monitoring populations of rodent reservoirs of zoonotic diseases [in German]. BundesgesundheitsblattGesundheitsforschung-Gesundheitsschutz 2014; 57: 511-518. 